\title{
Psicoterapia de la Psicosis: De la Persona en Riesgo al Paciente Crónico
}

\section{Psychotherapy of Psychosis: From the Subject at Risk to the Chronic Patient}

\author{
Nuria Esteve Díaz, Nuria Román Avezuela, Manuel González Moliner, Juan Carlos Fraile Fraile e \\ Ignacio García-Cabeza \\ HGU Gregorio Marañón-España
}

\begin{abstract}
Resumen. Los autores revisan las intervenciones psicoterapéuticas más utilizadas en pacientes psicóticos, describiendo por un lado aquellas intervenciones realizadas durante las fases tempranas de la enfermedad, las intervenciones psicoterapéuticas clásicas durante los periodos de estabilidad del paciente y finalmente aquellas relacionadas con la rehabilitación psicosocial del enfermo psicótico. Durante las fases tempranas se diferencia entre tratamientos psicoterapéuticos en pacientes de alto riesgo y los que ya padecen una psicosis franca. Se describe la eficacia y la forma de intervención actual de cada uno de los cuatro modelos psicoterapéuticos más convencionales: dinámico, cognitivo-conductual, familiar y grupal. Se hace un especial énfasis en la necesidad de intervenciones integradoras y orientadas a las necesidades de cada paciente. Por último, se describen las intervenciones psicosociales que han demostrado su eficacia, a saber: tratamiento comunitario asertivo, entrenamiento en habilidades sociales y programas de apoyo al empleo.

Palabras clave: psicosis, psicoterapia, intervención precoz, rehabilitación psicosocial.
\end{abstract}

\begin{abstract}
The authors review the psychotherapy interventions used most in psychotic patients, stressing, firstly, those conducted in the early phases of the disease, the most classical interventions during periods of stability, and, finally interventions related with psychosocial rehabilitation of the psychotic patient. Within the early phases of the disease, a distinction is made between high-risk subjects and those who have already had a first psychotic episode. The efficacy and the current intervention model in each of the most conventional psychotherapies: dynamic, cognitive-behavioral, family, and group therapies, are described. Special emphasis is placed on adaptation to the patient's needs in each intervention model. Finally, the psychosocial interventions that have demonstrated their efficacy are indicated, that is to say, assertive community therapy, social skills training and supported employment programs.Key words: psychosis, psychotherapy, early intervention, psychosocial rehabilitation.
\end{abstract}

\section{Introducción}

El abordaje psicofarmacológico del paciente esquizofrénico en muchos casos imprescindible para la disminución de los síntomas psicóticos, no supone un remedio para afrontar las vulnerabilidades, conflictos, problemas o tensiones interpersonales o

La correspondencia sobre este artículo dirigirla a Ignacio García Cabeza. Servicio de Psiquiatría I. HGU Gregorio Marañón. C/ Ibiza 43. 28009-Madrid. e-mail: igcabeza@ya.com biográficas, ni tampoco para analizar o modificar estilos perceptivos o mecanismos de defensa. Además, su uso generalizado nos ha mostrado también su falta de eficacia en determinados enfermos. Todo ello nos hace reflexionar sobre la necesidad de intervenciones psicoterapéuticas que palien las limitaciones de un abordaje exclusivamente farmacológico. En este sentido, la combinación de antipsicóticos con otros tipos de abordaje terapéuticos hace que ambos métodos se influyan y potencien (García 
Cabeza y González de Chávez, 2009; M González de Chávez, García Cabeza y Fraile, 1999).

Revisaremos a continuación las intervenciones psicoterapéuticas más utilizadas en pacientes psicóticos distinguiendo por un lado aquellas que se realizan en fases tempranas de la enfermedad, las intervenciones más clásicas en periodos de estabilidad y por último y brevemente intervenciones relacionadas con la rehabilitación psicosocial del enfermo psicótico.

\section{Intervención en fases tempranas}

La detección e intervención precoz en la esquizofrenia, así como el tratamiento de los primeros episodios psicóticos han despertado un gran interés a la vez que han generado no pocas dudas y controversias, motivado probablemente por la falta de conocimiento que en la actualidad existe sobre muchos aspectos de esta patología.

El auge en cuanto a número de estudios y programas de intervención precoz en psicosis se asienta en la evidencia de que la demora en el inicio del tratamiento en pacientes que han sufrido un primer episodio se asocia a peor respuesta al tratamiento farmacológico, mayor severidad de síntomas positivos, negativos y depresivos y peor funcionamiento global (Francey, et al., 2010; Marshall, et al., 2005; Perkins, $\mathrm{Gu}$, Boteva y Lieberman, 2005). Estudios de neuroimagen también indican que un periodo prolongado de enfermedad no tratada produce anormalidades estructurales cerebrales más pronunciadas (Francey, et al., 2010; Keshavan y Amirsadri, 2007).

Revisamos seguidamente las técnicas psicoterapéuticas eficaces empleadas en estos momentos de la enfermedad, diferenciando pacientes de riesgo, en los que todavía no se ha producido el debut de la sintomatología psicótica, de los que padecen un primer episodio.

\section{Intervenciones psicoterapéuticas en pacientes de alto riesgo}

La definición de sujetos de alto riesgo procede de numerosos estudios de investigación que detectan características clínicas que permiten identificar sujetos con gran probabilidad de desarrollar psicosis en el futuro. Tres son las definiciones más influyentes de este concepto.

La más extendida es la de "riesgo ultra-alto", desarrollada por el grupo de trabajo PACE (Personal Assessment and Crisis Evaluation) en Melbourne y que incluye la edad (entre 14 y 29 años) y criterios de al menos uno de los siguientes grupos: (1) Grupo de Síntomas Psicóticos Atenuados (haber experimentado síntomas durante el año previo que no alcanzan el umbral); (2) Grupo de Síntomas Psicóticos Breves Limitados e Intermitentes (en inglés BLIPS), que se corresponden a episodios psicóticos francos de menos de una semana de duración; (3) Grupo de Rasgo y Estado como Factor de Riesgo (tener un trastorno esquizotípico de la personalidad o un familiar de primer grado con un trastorno psicótico y haber sufrido durante el año previo un deterioro significativo en el funcionamiento global) (Yung, et al., 2003; Yung, Phillips, Yuen y Mc Gorry, 2004).

El grupo de Reconocimiento y Prevención de Hillside (Hillside-RAP) en Nueva York, en función de sus estudios de evolución, modifica estos criterios y plantea dos categorías: pacientes de "alto riesgo clínico" (CHR) y pacientes con "psicosis esquizofrenia-like" (SLP). Los primeros se clasifican como de alto riesgo clínico negativo (que cursan con síntomas negativos atenuados: aislamiento social, abulia y deterioro del rol o funcionamiento académico) y positivo (adolescentes con Síntomas Psicóticos Atenuados). El grupo esquizofrenia-like incluye a personas con síntomas psicóticos que no llegan a cumplir criterios DSM-IV para la esquizofrenia (Cornblatt, 2002)

Por último, en Europa el grupo alemán de Colonia define un cluster con los 10 "síntomas básicos" de mayor valor predictivo: interferencias en el pensamiento, perseveración, presión o bloqueos, alteración en el lenguaje receptivo (comprehensivo, no verbal), disminución de la capacidad para diferenciar entre ideas y percepciones o fantasía y recuerdos verdaderos, ideas ocasionales de referencia, desrealización, alteraciones perceptivas visuales o auditivas (Klosterkotter, Hellmich, Steinmeyer y Schultze-Lutter, 2001). 
Son escasos los estudios que se han llevado a cabo sobre la eficacia de una intervención psicoterapéutica en estos sujetos. La terapia cognitivo conductual es hasta la fecha la única cuya utilidad ha podido ser demostrada. En el estudio realizado por PACE, se comparan un grupo control referido a un programa de gestión de casos (psicoterapia de apoyo, terapia familiar, asistencia con alojamiento y programa de formación/empleo) con un grupo de tratamiento que además recibió dosis bajas de risperidona y terapia cognitivo-conductual modificada. Aunque la intervención farmacológica y psicológica retrasa la aparición de la psicosis, no demuestra que reduzca la incidencia ni tampoco existen diferencias en la tasa de transición ni el nivel de sintomatología o funcionamiento entre ambos grupos (Jane Edwards y McGorry, 2002; McGorry, et al., 2002; Phillips, et al., 2007).

El estudio EDIE (Early Detection and Intervention Evaluation Trial) en Reino Unido confronta terapia cognitiva y elementos de gestión de casos con un grupo control en donde sólo se utilizó esto último. Aunque es menor el número de pacientes en el grupo de intervención que desarrollan psicosis, no alcanza niveles significativos. El seguimiento a tres años sí encuentra diferencias en la transición a la psicosis cuando se controlan algunos síntomas cognitivos (incontrolabilidad de pensamientos no deseados y miedo al rechazo y criticismo) y, reduce significativamente la prescripción de antipsicóticos (Morrison, et al., 2007; Morrison, et al., 2004)

El OPUS danés mide la tasa de transición de personas con un trastorno esquizotípico de la personalidad a psicosis, encontrando que una intervención integradora retrasa o inhibe su aparición frente al tratamiento estándar (Nordentoft, et al., 2006).

En líneas generales, las conclusiones a las que podrían llevarnos estos estudios es que parece existir cierto beneficio en la detección e intervención en sujetos de alto riesgo. La combinación de tratamientos farmacológicos y psicológicos es la que mejores resultados obtiene, no siendo eficaces aquellas intervenciones que sólo incluían fármacos (McGlashan, et al., 2006).

\section{Intervenciones psicoterapéuticas en primeros episodios psicóticos}

El objetivo fundamental que se persigue a la hora de practicar intervenciones en estas primeras fases de la enfermedad es evitar la cronificación del paciente, para ello es sumamente importante reducir al máximo el periodo de la enfermedad sin tratar (PNT), poniendo en marcha intervenciones específicas lo antes posible. Los tratamientos psicológicos han demostrado ser de gran ayuda a la hora de potenciar los efectos de los farmacológicos, para trabajar sobre contenidos delirantes que de otro modo tienen un alto riesgo de permanecer imperturbables y para amortiguar el impacto que las propias experiencia psicóticas o algunos abordajes, como los ingresos hospitalarios, tienen en los pacientes. Aunque las guías nacionales e internacionales en general abogan por abordajes psicoterapéuticos que incluyan terapia cognitivo-conductual, intervenciones psicoeducativas y familiares, en general la puesta en marcha de programas integradores son las que han puesto de manifiesto mayor eficacia (Bravo et al, 2009).

La terapia cognitiva persigue entre otros objetivos que el paciente sea capaz de tener un mayor control sobre los síntomas psicóticos, educarle sobre la naturaleza de los mismos, que pueda identificar pensamientos negativos o molestos y que sepa en qué contextos surgen, así como aprender manejos alternativos ante situaciones estresantes y favorecer una pronta y adecuada recuperación (Bravo et al, 2009). En distintos estudios la terapia cognitiva mejora síntomas positivos persistentes, sobre todo en estadios precoces, alucinaciones auditivas, sentimientos de desesperanza, puede ayudar a mejorar la adaptación a la enfermedad, la adherencia al tratamiento, e incluso a reducir el abuso de sustancias (Penn, Waldheter, Perkins, Mueser y Lieberman, 2005; Scott, Chant, Andrews y McGrath, 2006; Tarrier, 2005).

No todos obtienen tan buenos resultados. En Australia, dentro del programa EPPIC, la Psicoterapia de Orientación Cognitiva para la Psicosis Temprana (COPE) sólo encuentra diferencias significativas en que existe mayor tendencia a la integración de la psicosis frente al grupo control que tiende 
al sealing-over (Jackson, et al., 2001). El Study of Cognitive Realignement Therapy in Early Schizophrenia (SoCRATES) realizado de forma específica en psicosis de inicio reciente y comparando terapia de apoyo, cognitiva conductual y tratamiento estándar, no encuentra diferencias con la terapia de apoyo salvo en la reducción de alucinaciones auditivas, aunque ambas son claramente superiores al tratamiento convencional (Tarrier, et al., 2004).

Otro aspecto que debe tenerse en cuenta en pacientes que han superado un primer episodio psicótico es la repercusión emocional que éste genera, son comunes los sentimientos de vergüenza, minusvalía, culpa, ansiedad social o baja autoestima y no es infrecuente que se asocien síntomas propios del trastorno por estrés post traumático. La intervención cognitivo conductual muestra una mejoría significativa de este tipo de síntomas (Jackson, et al., 2009; Mueser, et al., 2008). El estudio LifeSPAN australiano que incorpora terapia cognitiva breve en pacientes con primeros episodios e ideación suicida obtiene buenos resultados en la prevención suicida (Power, et al., 2003).

La aplicación de técnicas psicoeducativas debe ir enfocada tanto a la figura del paciente, como de los familiares. Se pretende aumentar el conocimiento que se tiene sobre la enfermedad, desmontando mitos y fomentando su aclaración. La información dada deberá ser revisada y actualizada cada cierto tiempo, adaptándose a los niveles culturales e intelectuales de todos los miembros. Es fundamental en estos primeros estadios tratar de desculpabilizar a los familiares e infundir esperanzas de futuro (Bravo et al, 2009). Algunos estudios encuentran una reducción hasta de un $20 \%$ en las recaídas cuando se incluyen a familiares en el tratamiento (Francey, et al., 2010).

La intervención familiar aborda las dinámicas intrafamiliares, recoge las explicaciones que cada familiar da al comportamiento del paciente, se tratan de solventar los conflictos existentes, se analizan las emociones más comunes que aparecen tras los primeros episodios, se da soporte e información, entre otras intervenciones (Bravo et al, 2009). La intervención familiar acorta el tiempo de hospitalización y evita recaídas, como en cualquier otro momento de la evolución de la enfermedad (Dingemans, et al., 1996; Wang, Li y Phillips, 1994).

McGorry, en su libro "Intervención precoz en la psicosis", explica los principios que deben tenerse en cuenta en el tratamiento inicial de un primer episodio psicótico. Aunque señala la importancia del tratamiento farmacológico y los logros obtenidos mediante la terapia cognitivo conductual, todo debe formar parte de un tratamiento integrado de los aspectos "biológicos, sociales y psicológicos". Esta terapia integral debe adaptar de forma flexible los objetivos a las necesidades del paciente y tener en cuenta las distintas fases de la enfermedad. La intervención biológica principal es el tratamiento farmacológico y las intervenciones psicológicas tienen como objetivo explicar al paciente en qué consiste la enfermedad, dar apoyo y confianza de cara a la recuperación, y explicar por qué es preciso tomar la medicación y cuáles son los efectos de ésta. Los aspectos sociales deben incluir el trabajo con la familia y la red social del paciente así como la ayuda al paciente en la resolución de problemas cotidianos (Edwards y McGorry, 2004).

Se han llevado a cabo varios estudios con el objetivo de evaluar las intervenciones de carácter integrador en primeros episodios psicóticos. El OPUS danés compara pacientes que reciben un tratamiento convencional, psicodinámico y tratamiento integrado (tratamiento asertivo, psicoeducación y habilidades sociales). Los resultados del seguimiento los dos primeros años demuestran que el tratamiento integrador facilita una mayor recuperación tanto sintomática como funcional, así como menos carga familiar (Petersen, et al., 2005), a los cinco años las diferencias significativas quedan reducidas a una mayor autonomía y un menor número de hospitalizaciones (Bertelsen, et al., 2009). El también randomizado LEO (Lambeth Early Onset) que incluye tanto primeros como segundos episodios si no se recogieron en un primer momento encuentra, desde una intervención asertiva, que pacientes con tratamiento específico tienen menos recaídas, rehospitalizaciones y abandonos, mejor adherencia al tratamiento y dedican más tiempo a actividades de formación o con fines profesionales y establecen o reestablecen mejores relaciones sociales (Craig, et al., 2004). También desde una perspectiva integradora el pro- 
yecto Parachute, encuentra diferencias significativas en el funcionamiento global durante el primer año incluso cuando se compara con un tratamiento convencional de alta calidad (Cullberg, Levander, Holmqvist, Mattsson y Wieselgren, 2002), y a los tres años una evolución significativamente mejor tanto a nivel sintomático como funcional, reduciendo los costes del tratamiento, probablemente debido al menor número de hospitalizaciones (Cullberg, et al., 2006). El modelo finlandés adaptado a las necesidades del paciente ofrece unos espléndidos datos en el seguimiento a cinco años con menos psicopatología, menos hospitalizaciones, mejor ajuste y menos incapacidades (Alanen, 2003).

\section{Intervenciones clásicas en la psicoterapia de la psicosis: estado actual}

Aunque por cuestiones didácticas vamos a describir individualmente cada uno de los cuatro grandes enfoques psicoterapéuticos (dinámico, cognitivo conductual, familiar y grupal), la tendencia en nuestros días, como al fin y al cabo sucede en el resto de las patologías, es hacia enfoques integradores, encontrando cada vez más puntos en común entre todos los abordajes que diferencias (Alanen, 2003). En el caso de las psicosis no cabe duda que las intervenciones precoces son pioneras, como hemos comentado, pero también son cada vez más utilizadas en otros momentos de la enfermedad, sobre todo en aquellos países donde mayor es el esfuerzo en el tratamiento psicológico. Cada uno de dichos tratamientos presenta sus peculiaridades: por ejemplo, en el modelo finlandés adaptado a las necesidades, priman intervenciones individuales y familiares (Alanen, 2003); en el sueco, las intervenciones comunitarias (Cullberg, et al., 2002; Cullberg y McGorry, 2006); en el danés, las intervenciones asertivas y psicosociales (Petersen, et al., 2005) o tendencias más cognitivistas en los países anglosajones (McGorry, et al., 2002).

En los últimos años las terapias de orientación dinámica han sufrido distintas críticas que han limitado su uso. Comenzando con el estudio de Boston donde no se encuentran diferencias entre psicoterapias de apoyo y orientadas al insight (Gunderson,
1984) y, sobre todo, a raíz de la publicación en 1998 de las primeras recomendaciones PORT (Schizophrenia Patient Outcome Research Team) para el tratamiento de la esquizofrenia, donde son excluidas como forma eficaz de tratamiento para la psicosis (Lehman y Steinwachs, 1998). Previamente Mc Glashan ya centra la aproximación psicodinámica en el tiempo presente más que en el pasado, combinando estrategias de apoyo e introspección (Mc Glashan, 1994).

Coincidiendo con la publicación de las primeras recomendaciones PORT se crea un grupo de estudio de las terapias dinámicas que pone énfasis en una serie de puntos necesarios para que éstas sean aplicables y eficaces: exclusión de las aproximaciones más clásicas, incidiendo en el presente sobre el pasado; utilizando las interpretaciones con extrema cautela, con objetivos concretos como afianzar el proceso de individuación, la estabilización de los límites del Yo y la identidad e integrando las experiencias psicóticas y, llevadas a cabo siempre por terapeutas experimentados (Bachmann, Resch y Mundth, 2003). En general estas intervenciones se esfuerzan en entender al paciente en su contexto biográfico integrando las vivencias psicóticas, afirmando los límites del Yo y ayudando al proceso de individuación (García Cabeza y González de Chávez, 2009) y existen algunas evidencias sobre su eficacia (Mojtabai, Malaspina y Susser, 2003), sobre todo en aquellos pacientes más estables (Hauff, et al., 2002).

Los abordajes cognitivo-conductales son los que más han evolucionado en los últimos tiempos, desde posturas centradas en el abordaje de los síntomas (positivos, negativos, depresión, ansiedad) hasta modelos globales centrados en la persona como en la terapia personal de Hogarty (Hogarty, Flescher, Ulrich y Carter M, 2004), con un enfoque orientado a la respuesta emocional y cognitiva ante el estrés para dotar al individuo de estrategias de afrontamiento o, la centrada en la persona de Chadwick (Chadwick, 2006).

Numerosas publicaciones apoyan la utilidad de los abordajes cognitivo-conductuales. Entre ellas cabe destacar el metaanálisis de Rector (Rector y Beck, 2001)), la revisión de artículos de Dickerson (Dickerson, 2004)) y las recomendaciones de la Revisión de Programas Psicosociales (Dixon, 
Dickerson y Bellack, 2009). Estas últimas abogan por la utilidad de la terapia cognitivo conductual tanto en el tratamiento de síntomas negativos como positivos resistentes a psicofarmacoterapia. Sin embargo, en un reciente metaanálisis sobre estudios de intervenciones específicas, cuando se compara la terapia cognitiva conductual con abordajes psicoeducativos no detecta diferencias en cuanto a prevención de recaídas y mejoría de síntomas negativos y sí en los síntomas positivos (Lynch, Laws y McKenna, 2010)

En cualquier caso y como ya hemos comentado las terapias cognitivas han sufrido una importante evolución y aunque los síntomas siguen siendo un importante foco de tratamiento, Turkington y cols. definen cuáles deben ser las etapas clave que la terapia cognitivo-conductual debe incluir en el momento actual: 1) desarrollo de una alianza terapéutica basada en la perspectiva del paciente; 2) desarrollo de explicaciones alternativas a los síntomas de la esquizofrenia; 3) reducir el impacto de síntomas positivos y negativos; 4) ofrecer alternativas al modelo médico para facilitar la adherencia al tratamiento (Turkington, Kingdon y Weiden, 2006). Chadwick va un paso más allá y describe un modelo de intervención cognitiva que define como basado en la persona donde la relación terapéutica, entendida desde un punto de vista rogeriano, ocupa un lugar principal en el proceso terapéutico; incluye también la meditación con conciencia plena y define el insight metacognitivo, para conceptualizar el estrés y articular los procesos de cambio y; el concepto de self como esquema y proceso. Abandona elementos clásicos de la terapia cognitiva y define la exploración terapéutica de la denominada "Zona de Futuro Desarrollo" donde con el apoyo de un terapeuta implicado y experimentado, el paciente tolera el estrés, desarrolla insight metacognitivo y alcanza la auto aceptación a través del desarrollo y fortalecimiento futuro en cuatro dominios, fuentes de estrés (el significado de los síntomas, la relación con la experiencia interna, el esquema y el self simbólico) (Chadwick, 2009).

Al igual que las terapias cognitivo-conductuales las terapias familiares han sufrido una enorme evolución, aunque en este caso a lo largo de décadas. Los modelos de intervención psicoanalíticos se vieron rápidamente desplazados por modelos sistémicos, más breves y que permiten el trabajo en equipo, más seguro y gratificante, primordial dentro del modelo finlandés adaptado a las necesidades (Lehtinen, 1993). Hoy en día los modelos que se consideran eficaces son intervenciones prolongadas, más de seis meses, que van más allá de la mera psicoeducación y combinan con ésta, apoyo emocional, intervención en crisis y estrategias de afrontamiento de los síntomas de la enfermedad y problemas relacionados, reduciendo la tasa de recaídas, rehospitalizaciones y disminuyendo la carga familiar, (Dixon, et al., 2009; Patterson y Leeuwenkamp, 2008; Pharoa, 2007).

Comparada con las anteriores las terapias de grupo, aunque eficaces en la psicosis (Kanas, 1996; Stone, 1996), han sido menos estudiadas entre otras causas por la dificultad intrínseca en la investigación de las mismas., las limitaciones en cuanto a la teorización y conceptualización de la complejidad grupal, la gran variedad de grupos y la singularidad de cada uno de ellos (González de Chávez, 2008). De hecho, la mayoría de estudios randomizados los encontramos en la aplicación grupal de intervenciones cognitvas, de habilidades sociales o el Tratamiento Integrado (Tost, et al., 2008). Sin embargo, pensamos que sólo cuando se utiliza un abordaje ecléctico y adaptado a las necesidades se obtiene el verdadero beneficio de la psicoterapia grupal. Así nosotros proponemos un modelo flexible e integrador, que partiendo del ambiente socializador que supone el grupo, del apoyo, consejo y técnicas de afrontamiento del "aquí y ahora" permita cuando el grupo adquiere la suficiente cohesión, los pacientes están estabilizados, con buena tolerancia a la de ansiedad y viviendo escasas experiencias psicóticas, la utilización de las mismas con fines terapéuticos, llegando en las fases más avanzadas a un abordaje dinámico de la problemática del paciente, manteniendo siempre presentes estrategias de apoyo, afrontamiento e interpersonales (García Cabeza, 2008; García Cabeza y González de Chávez, 2009; M González de Chávez, et al., 1999)

\section{El paciente psicótico en la comunidad}

El mantenimiento del paciente psicótico en el ámbito ambulatorio y comunitario es un objetivo 
principal en su tratamiento. Aunque de un modo algo artificioso hemos distinguido aquí las ya mencionadas intervenciones psicoterapéuticas clásicas de otras intervenciones psicosociales más ligadas a la rehabilitación. Haremos una breve mención, ya que merecen un capítulo aparte, aquellas que han demostrado fehacientemente su eficacia y que aparecen citadas en todas las recientes recomendaciones del tratamiento de la esquizofrenia: tratamiento comunitario asertivo, entrenamiento en habilidades sociales, rehabilitación cognitiva y empleo (Dixon, et al., 2009; Patterson y Leeuwenkamp, 2008; Silverstein, Spaulding y Menditto, 2006)

Definíamos como artificiosa esta división porque, por ejemplo las técnicas cognitivo-conductuales forman parte de algunos de los programas integrados más conocidos para el entrenamiento en habilidades sociales y de rehabilitación cognitiva: el "Social and Independent Living Skills" (Liberman, Mueser y Wallace, 1986), el "Integrated Psychological Therapy" (IPT) (Brenner, Hodel, Roder y Corri-gan, 1992), el Cognitive Enhacement Therapy" (CET) (Hogarty, et al., 2004) o el "Cognitive Adaptation
Training" (CAT) (Velligan, et al., 2000). Además cualquier intervención comunitaria de rehabilitación psicosocial incluye programas psicoeducativos para pacientes y familiares. En general psicoterapias y rehabilitación comparten muchos objetivos con lenguajes, énfasis y contextos diferentes (Harding y McCrory, 2008).

Sea como fuere, centrándonos en abordajes rehabilitadores dirigidos básicamente a la funcionalidad encontramos que la Terapia Asertiva Comunitaria disminuye el uso de sustancias, la hospitalización y posiblemente también el riesgo de exclusión social (Dixon, et al., 2009; Patterson y Leeuwenkamp, 2008). 25 años de práctica y numerosos estudios controlados avalan la eficacia del entrenamiento en habilidades sociales (Benton y Schroeder, 1990; Liberman y Kopelowicz, 2005) y el apoyo al empleo se ha mostrado eficaz en 16 estudios randomizados (Killackey, 2009). Por último y aunque es evidente la eficacia de la rehabilitación cognitiva en la mejoría de resultados en tests neuropsicológicos, no lo es tanto en lo referido a su capacidad para incrementar la funcionalidad del paciente en el día a día (Patterson y Leeuwenkamp, 2008).

\section{Extended Summary}

The psychopharmacological approach to the schizophrenic patient, although useful, or even essential in many cases in order to reduce psychotic symptoms, is not in itself a remedy to cope with their vulnerabilities, conflicts, problems or interpersonal or biographical tensions, nor does it serve to analyze or modify perceptive styles or defense mechanisms. Furthermore, its generalized use has also shown lack of efficacy in some patients. All of this leads us to reflect on the need for psychotherapeutic interventions that address the limitations of an exclusively pharmacological approach. In this sense, the combination of antipsychotics with other types of therapeutic approaches mutually influences and strengthens both methods.

The psychotherapeutic interventions most used in psychotic patients (as distinguished from those carried out in the early phases of the disease), the more classical interventions in periods of stability and finally interventions related with psychosocial rehabilitation of the psychotic patient, are all reviewed in the following article.

Within the effective psychotherapeutic techniques used in the early phases of the disease, we can distinguish those interventions in patients at risk in which no clear onset of the psychotic symptoms has occurred and those psychotherapeutic modalities used in individuals who have already suffered a first psychotic episode.

The definition of at high-risk subjects comes from the many research studies, the most extended of which is that developed by the Melbourne PACE group. This study included age (from 14 to 29 years old) and criteria from at least one of the following groups: (1) Attenuated Psychotic Symptoms Group (having experienced symptoms that did not reach the threshold during the previous year); (2) Brief Limited Intermittent Psychotic Symptoms Group (BLIPS, which corresponds to frank psychotic episodes lasting at least one week); (3) State plus Trait 
group as Risk Factor (having a schizotypal personality disorder or a first degree family member with a psychotic disorder and having suffered significant deterioration in global functioning in the previous year). Other definitions come from the Hillside Recognition and Prevention program and the German Colonial policy.

Few studies have been conducted on the efficacy of psychotherapy intervention in these subjects. Up to now, cognitive behavioral therapy has been the only therapy where utility could be demonstrated, with no efficacy being found in those interventions that only include drugs. Along general lines, there seems to be some benefit in the detection and intervention in high-risk subjects, although integrated and flexible programs aimed at early detection and intervention, attending to each case specifically, need to be created.

Psychological therapies have been shown to be very helpful when reenforcing the effects of pharmacological treatments. They work on delusional contents that otherwise would have a high risk of remaining undisturbed over time and so soften the impact that psychotic experiences, or indeed some approaches, such as hospitalization, have on the patients. The national and international guidelines support psychotherapeutic approaches that include cognitive-behavioral therapy, psychoeducational and family interventions, although in general, the initiation of integrated programs is more effective.

Cognitive therapy aims, among other things, to enable the patient to have greater control over their psychotic symptoms, educating them about their own nature. It also aims to help the patient identify negative or unpleasant thoughts and to know the context in which they arise, to learn alternative managements when there are stressful situations, and favoring fast and adequate recovery as much as possible. Cognitive therapy reduces persistent positive symptoms, auditory hallucinations and feelings of hopelessness. It helps to improve adaptation to the disease, treatment adherence, and even to reduce substance abuse. It also shows a significant improvement in post-traumatic stress symptoms and suicidal ideation.

The application of psychoeducational techniques should be focused both on the patient and their family members. The techniques aim to increase their knowledge about the disease, dismantle myths and encourage their clarification. The information given should be reviewed and updated periodically, adapting it to the cultural and intellectual levels of all the members. It is essential in the first stages to try to free the family members from blame and to inspire hope for the future. Some studies have found that relapses decrease up $20 \%$ when family members are included in the treatment.

The family intervention deals with intra-family dynamics, gathering the explanations that each family member gives for the patient's behavior, and tries to solve the existing conflicts. It analyzes the most common emotions that appear after the first episodes, gives support and information, among many other interventions. Family intervention shortens hospitalization time and avoids relapses.

McGorry demonstrated the principles that should be taken into account in initial treatment of a first psychotic episode. Although he emphasized the importance of drug treatment and the achievements obtained through cognitive-behavior therapy, this should all form a part of an integrated treatment of the "biological, social and psychological" aspects. This integral therapy should adapt the objectives flexibly to the patient's needs and take the different phases of the disease into account. The main biological intervention is pharmacological treatment for symptomatic improvement. The aim of the psychological interventions would be to explain what the disease consists of to the patient, giving support and confidence regarding recovery and explaining why it is necessary to take the medication and what its effects are. The social aspects should include working with the family and the social network of the patient and helping the patient in daily problem solving.

Although for didactic reasons we are going to describe each one of the four large psychotherapeutic approaches individually (dynamic, cognitivebehavioral, family and group approaches), the present tendency is towards integrated approaches. More and more points are being found in common than differences between all of the approaches.

In recent years, several criticisms have been aimed at dynamic orientation therapies, which have 
limited use of this type of therapy. In particular we may mention the publication of the first Patient Outcome Research Team (PORT) recommendations for the treatment of schizophrenia, in which dynamic therapies are excluded as an effective treatment form. Coinciding with these recommendations, PORT created a study group for dynamic therapies, which stressed a series of points that are necessary if these therapies are to be appropriate and effective. These included exclusion of the more classical approaches, stressing the present over the past, using the interpretations with extreme caution, and with specific objectives such as reinforcing the process of individuation, stabilization of the limits of the self and the identity and integrating the psychotic experiences. These always had to be performed by expert therapists. In general, these interventions are intended for understanding the patient within his/her biographical context, integrating the psychotic experiences, reinforcing the limits of the self and helping in the individuation process. Indeed, there is recent evidence of its efficacy, and even more so in stabilized patients.

Cognitive-behavioral approaches are those that have evolved the most in recent years, going from positions focused on the approach of symptoms, moving towards global models focused on the person. Many publications support the utility of cognitive-behavior approaches. In 2006, Turkington et al. defined which key stages should be included in cognitive-behavior therapy at present: 1) developing a therapeutic alliance based on the patient's perspective; 2) developing alternative explanations of schizophrenia symptoms; 3) reducing the impact of the positive and negative symptoms; 4) offering alternatives to the medical model to facilitate medication adherence.

As with cognitive-behavior therapies, family therapies have also undergone enormous evolution. Nowadays, the models that have been shown to be the most effective are those of prolonged interventions, more than six months, which go beyond mere psychoeducation and which combine emotional support, intervention in crisis and coping strategies for the disease symptoms and related problems, thus reducing the relapse and rehospitalization rate, and decreasing the burden on the family.
Compared with the therapies already described, group therapies, although effective in psychosis, have been studied less. In fact, most of the randomized studies found are about group application of cognitive interventions, social skills or Integrated Treatment. However, we consider that the true benefit of group psychotherapy is only obtained when an eclectic, flexible approach that is adapted to needs is adopted.

We therefore propose a flexible and integrated model. This model uses the socializing setting provided by the group, which gives support, advice and coping techniques in the "here and now." When the group has acquired sufficient cohesiveness, when the patients are stabilized, have good tolerance to anxiety and have few psychotic experiences, this model permits the use of these techniques with therapeutic purposes. In the final phases, the model reaches a dynamic approach to the patient's problems, though it always maintains the support, coping and interpersonal strategies.

In summary, with regard to the psychosocial approach, we know that Assertive Communitarian Therapy decreases the use of drugs, hospitalization and possibly also the risk of social exclusion, that 25 years of psychiatric rehabilitation and many controlled studies support the efficacy of social skills training and that employment support has been shown to be effective in 16 randomized studies. Cognitive rehabilitation techniques show improvement in the neuropsychological test results, although to a lesser extent in those regarding the patient's day-to-day functionality.

\section{Referencias}

Alanen, Y. (2003). La esquizofrenia: sus orígenes y su tratamiento adaptado a las necesidades del paciente. Madrid: Fundación para la Investigación y Tratamiento de la Esquizofrenia y otras Psicosis.

Bachmann, S., Resch, F. y Mundth, C. ( 2003). Psycological treatments for psychosis: history and overview. Journal of American Academy of Psychoanalysis and Dynamic Psychiatry, 31, 155-176. 
Benton, M. K. y Schroeder, H. E. (1990). Social skills training with schizophrenics: a meta-analytic evaluation. Journal of Consulting and Clinical Psychology, 58, 741-747.

Bertelsen, M., Jeppesen, P., Petersen, L., Thorup, A., Ohlenschlaeger, J., Le Quach, P., et al. (2009). Course of illness in a sample of 265 patients with first-episode psychosis-five-year follow-up of the Danish OPUS trial. Schizophrenia Research, 107(2-3), 173-178.

Bravo MF, V. O., San Emeterio M, Cid J, Ibañez V, González Cases J, Fernández Liria A. . (2009). Consenso sobre la Atención Temprana a la psicosis de la Asociación Española de Neuropsiquiatría. Paper presented at the Revista de la Asociación Española de Neuropsiquiatría, Madrid.

Brenner, H. D., Hodel, B., Roder, V. y Corrigan, P. (1992). Treatment of cognitive dysfunctions and behavioral deficits in schizophrenia. Schizophrenia Bulletin, 18, 21-26.

Cornblatt, B. A. (2002). The New York high risk project to the Hillside recognition and prevention (RAP) program. American Journal of Medical Genetics, 114, 956-966.

Craig, T. K., Garety, P., Power, P., Rahaman, N., Colbert, S., Fornells-Ambrojo, M., et al. (2004). The Lambeth Early Onset (LEO) Team: randomised controlled trial of the effectiveness of specialised care for early psychosis. $B M J$, 329(7474), 1067.

Cullberg, J., Levander, S., Holmqvist, R., Mattsson, M. y Wieselgren, I. M. (2002). One-year outcome in first episode psychosis patients in the Swedish Parachute project. Acta Psychiatrica Scandinavica, 106, 276-285.

Cullberg, J., Mattsson, M., Levander, S., Holmqvist, R., Tomsmark, L., Elingfors, C., et al. (2006). Treatment costs and clinical outcome for first episode schizophrenia patients: a 3-year followup of the Swedish 'Parachute Project' and Two Comparison Groups. Acta Psychiatrica Scandinavica, 114, 274-281.

Cullberg, J. y McGorry, P. D. (2006). Psychoses: An integrative perspective. London; New York: Routledge.

Chadwick, P. (2006). Person-based cognitive therapy for distressing psicosis. Wiley: Chichester.
Chadwick, P. (2009). Psicoterapia cognitiva basada en la persona para la psicosis perturbadora. Madrid: Fundación para la Investigación y Tratamiento de la Esquizofrenia y otras Psicosis.

Dickerson, F. B. (2004). Update on cognitive behavioural psychotherapy for schizophrenia: review of recent studies. Journal of Cognitive Psychotherapy: An International Quarterly, 18, 189-205.

Dingemans, P., Van der Does, J., Nugter, A., Scholte, P., Lenior, R. y Goldstein, M. (1996). Treatment, expressed emotion and relapse in recent schizophrenic disorders. Psychological Medicine, 26, 333-342.

Dixon, L. B., Dickerson, F. y Bellack, A. S. (2009). The 2009 Schizophrenia PORT Psychosocial Treatment Recommendations and Summary Statements. Schizophrenia Bulletin, 36, 48-70.

Edwards, J. y McGorry, P. D. (2004). La intervención precoz en la psicosis: Guía para la creación de servicios de intervención precoz en la psicosis. Madrid: Fundación para la investigación y el tratamiento de la esquizofrenia y otras psicosis.

Francey, S. M., Nelson, B., Thompson, A., Parker, A. G., Kerr, M., Macneil, C., et al. (2010). Who needs antipsychotic medication in the earliest stages of psychosis? A reconsideration of benefits, risks, neurobiology and ethics in the era of early intervention. Schizophrenia Research, 119, 1-10.

García Cabeza, I. (2008). Evolución de la psicoterapia en la esquizofrenia. Revista de la Asociación Española de Neuropsiquiatría, 101, 9-25.

García Cabeza, I. y González de Chávez, M. (2009). Insight and therapeutic factors in group psychotherapy for schizophrenic outpatients Psychosis, 1, 134-144.

González de Chávez, M. (2008). Abordajes psicoterapéuticos de las psicosis esquizofrénicas: historia, desarrollo y perspectivas. Madrid Fundación para la Investigación y Tratamiento de la Esquizofrenia y otras Psicosis.

González de Chávez, M., García Cabeza, I. y Fraile, J. (1999). Dos grupos psicoterapéuticos de pacientes esquizofrénicos: hospitalizados y ambulatorios. Revista de la Asociación Española de Neuropsiquiatría, 72, 573-586.

Gunderson, J. G. (1984). Effects of psycotherapy in schizophrenia: comparative outcome of two 
forms of treatment. Schizophrenia Bulletin, 10, 564-598.

Harding, C. y McCrory, D. (2008). Relación entre abordajes psicoterapéuticos y la rehabilitación psiquiátrica en pacientes con enfermedades mentales graves y persistentes: una llamada hacia estrategias más integradoras. En Y. Alanen, M. González de Chávez, A. Silver y B. Martindale (Eds.), En Abordajes psicoterapéuticos de las psicosis esquizofrénicas. Historia, desarrollo y perspectivas. Madrid: Fundación para la investigación y tratamiento de la esquizofrenia y otras psicosis. Hauff, E., Varvin, S., Laake, P., Melle, I., Vaglum, P. y Friis, S. (2002). Inpatient psychotherapy copmpared with usual care for patients who have schizophrenia psychoses. Psychiatric Services, 53, 471-473.

Hogarty, G., Flescher, S., Ulrich, R. y Carter M, G. D., Pogue-Geile M., Keshavan M., Cooley S., DiBarri A. L., Garret A., Parepally H. y Zoretich R. (2004). Cognitive enhancement therapy for schizophrenia: effects of a 2-years randomised trial on cognition and behaviour. Archives of General Psychiatry, 61, 866-876.

Jackson, C., Trower, P., Reid, I., Smith, J., Hall, M., Townend, M., et al. (2009). Improving psychological adjustment following a first episode of psychosis: a randomised controlled trial of cognitive therapy to reduce post psychotic trauma symptoms. Behavaviour Research Therapy, 47(6), 454-462.

Jackson, H., McGorry, P., Henry, L., Edwards, J., Hulbert, C., Harrigan, S., et al. (2001). Cognitively oriented psychotherapy for early psychosis (COPE): a 1-year follow-up. British Journal Clinical Psychology, 40 (Pt 1), 57-70.

Kanas, N. (1996). Group therapy for schizophrenic patients. Washington: American Psychiatric Press, Inc.

Keshavan, M. S. y Amirsadri, A. (2007). Early intervention in schizophrenia: current and future perspectives. Current Psychiatry Reports, 9, 325-328.

Killackey, E. (2009). Psychosocial and psychological interventions in early psychosis: essential elements for recovery. Early interventions in Psychiatry, 3, 17-21.

Klosterkotter, J., Hellmich, M., Steinmeyer, E. M. y
Schultze-Lutter, F. (2001). Diagnosing schizophrenia in the initial prodromal phase. Archives of General Psychiatry, 58, 158-164.

Lehman, A. F. y Steinwachs, D. M. (1998). Translating research into practice: the Schizophrenia Patient Outcomes Research Team (PORT) treatment recommendations. Schizophrenia Bulletin, 24, 1-10.

Lehtienen, K. (1993). Need-adapted treatment of schizophrenia: a five-years follow-ap from the Turku Project. Acta Psychiatrica Scandinava, 87, 96-101.

Liberman, R. P. y Kopelowicz, A. (2005). Psychiatric rehabilitation Kaplan and Sadock's comprehensive textbook of Psychiatry. Nueva York: Lippincott Williams and Wilkins.

Liberman, R. P., Mueser, K. T. y Wallace, C. J. (1986). Social skills training for schizofrenic individual at risk of relapse. American Journal of Psychiatry, 143, 523-526.

Lynch, D., Laws, K. y McKenna, P. J. (2010). Cognitive behavioural therapy for major psychiatric disorder: Does it really work? A meta-analytical review of well-controlled trials. Psycological Medicine 40, 9-24.

Marshall, M., Lewis, S., Lockwood, A., Drake, R., Jones, P. y Croudace, T. (2005). Association between duration of untreated psychosis and outcome in cohorts of first-episode patients: a systematic review. Archives of General Psychiatry, 62, 975-983.

McGlashan, T. H. (1994). What has become of the psychotherapy of schizophrenia. Acta Psychiatrica Scandinava, 90 (supl. 384), 147-152.

McGlashan, T. H., Zipursky, R. B., Perkins, D., Addington, J., Miller, T., Woods, S. W., et al. (2006). Randomized, double-blind trial of olanzapine versus placebo in patients prodromally symptomatic for psychosis. American Journal of Psychiatry, 163, 790-799.

McGorry, P. D., Yung, A. R., Phillips, L. J., Yuen, H. P., Francey, S., Cosgrave, E. M., et al. (2002). Randomized controlled trial of interventions designed to reduce the risk of progression to firstepisode psychosis in a clinical sample with subthreshold symptoms. Archives of General Psychiatry, 59, 921-928. 
Mojtabai, R., Malaspina, D. y Susser, E. (2003). The concept of population prevention: application to schizophrenia. Schizophrenia Bulletin, 29, 791801.

Morrison, A. P., French, P., Parker, S., Roberts, M., Stevens, H., Bentall, R. P., et al. (2007). Threeyear follow-up of a randomized controlled trial of cognitive therapy for the prevention of psychosis in people at ultrahigh risk. Schizophrenia Bulletin, 33, 682-687.

Morrison, A. P., French, P., Walford, L., Lewis, S. W., Kilcommons, A., Green, J., et al. (2004). Cognitive therapy for the prevention of psychosis in people at ultra-high risk: randomised controlled trial. British Journal of Psychiatry, 185, 291-297.

Mueser, K. T., Rosenberg, S. D., Xie, H., Jankowski, M. K., Bolton, E. E., Lu, W., et al. (2008). A randomized controlled trial of cognitive-behavioral treatment for posttraumatic stress disorder in severe mental illness. Journal of Consulting Clinical Psycholy, 76, 259-271.

Nordentoft, M., Petersen, L., Jeppesen, P., Thorup, A. A., Abel, M. B., Ohlenschlaeger, J., et al. (2006). [OPUS: a randomised multicenter trial of integrated versus standard treatment for patients with a first-episode psychosis - secondary publication]. British Journal of Psychiatry, 168, 381384.

Patterson, T. L. y Leeuwenkamp, O. R. (2008). Adjunctive psychosocial therapies for the treatment of schizophrenia. Schizophrenia Research, 100, 108-119.

Penn, D. L., Waldheter, E. J., Perkins, D. O., Mueser, K. T. y Lieberman, J. A. (2005). Psychosocial treatment for first-episode psychosis: a research update. American Journal of Psychiatry, 162, 2220-2232.

Perkins, D. O., Gu, H., Boteva, K. y Lieberman, J. A. (2005). Relationship between duration of untreated psychosis and outcome in first-episode schizophrenia: a critical review and meta-analysis. American Journal of Psychiatry, 162(10), 1785-1804.

Petersen, L., Nordentoft, M., Jeppesen, P., Ohlenschaeger, J., Thorup, A., Christensen, T. O., et al. (2005). Improving 1-year outcome in first- episode psychosis: OPUS trial. British Journal of Psychiatry, Suppl, 48, 98-103.

Pharoa, F. (2007). Intervención familiar para la esquizzofrenia (Revisión Cochrane traducida). Oxford: La bibiloteca Cochrane Plus.

Phillips, L. J., McGorry, P. D., Yuen, H. P., Ward, J., Donovan, K., Kelly, D., et al. (2007). Medium term follow-up of a randomized controlled trial of interventions for young people at ultra high risk of psychosis. Schizophrenia Research, 96, 25-33.

Power, P. J., Bell, R. J., Mills, R., Herrman-Doig, T., Davern, M., Henry, L., et al. (2003). Suicide prevention in first episode psychosis: the development of a randomised controlled trial of cognitive therapy for acutely suicidal patients with early psychosis. Australian and New Zealand Journal of Psychiatry, 37, 414-420.

Rector, N. A. y Beck, A. T. (2001). Cognitive behavioural therapy for schizophrenia: an empirical review. Journal of Nervous and Mental Disease, 189, 278-287.

Scott, J., Chant, D., Andrews, G. y McGrath, J. (2006). Psychotic-like experiences in the general community: the correlates of CIDI psychosis screen items in an Australian sample. Psychological Medicine, 36, 231-238.

Silverstein, S. M., Spaulding, W. D. y Menditto, A. (2006). Schizophrenia (advances in psychoterapy-evidence-based practice). Göttingen: Hogreffe\&Huber.

Stone, W. (1996). Group psychotherapy for people with chronic mental illness. New York: The Guilford Press.

Tarrier, N. (2005). Cognitive behaviour therapy for schizophrenia - a review of development, evidence and implementation. Psychotherapy and Psychosomatics, 74, 136-144.

Tarrier, N., Lewis, S., Haddock, G., Bentall, R., Drake, R., Kinderman, P., et al. (2004). Cognitive-behavioural therapy in first-episode and early schizophrenia. 18-month follow-up of a randomised controlled trial. British Journal of Psychiatry, 184, 231-239.

Tost, L., Hernández, A., de Sotomayor, C., Rodríguez Pulido, F., Perestelo Pérez, L., Pérez Ramos, J., et al. (2008). Psicoterapias de grupo como técnica terapéutica en personas con esquizofrenia. 
Madrid: Plan Nacional para el SNS del MSC.

Turkington, D., Kingdon, D. y Weiden, P. J. (2006). Cognitive Behavior therapy for schizophrenia. American Journal of Psychiatry, 163, 365-373.

Velligan, D. I., Bow-Thomas, C. C., Huntzinger, C., Ritch, J., Ledbetter, N., Prihoda, T. J., et al. (2000). Randomized controlled trial of the use of compensatory strategies to enhance adaptive functioning in outpatients with schizophrenia. American Journal of Psychiatry, 157, 1317-1323.

Wang, M., Li, J. y Phillips, M. (1994). Ramdomized-control trial of family intervention for first-episode male schizophrenic patients: an 18month study in Suzhou, Jiangsu. British Journal of Psychiatry 165(supl 24), 96-102.

Yung, A. R., Phillips, L. J., Yuen, H. P., Francey, S. M., McFarlane, C. A., Hallgren, M., et al. (2003). Psychosis prediction: 12-month follow up of a high-risk ("prodromal") group. Schizophrenia Research, 60, 21-32.

Yung, A. R., Phillips, L. J., Yuen, H. P. y McGorry, P. D. (2004). Risk factors for psychosis in an ultra high-risk group: psychopathology and clinical features. Schizophrenia Research, 67, 131-142.

Manuscrito recibido: 04/09/2010

Revisión recibida: 23/09/2010

Manuscrito aceptado: 28/09/2010 